\title{
Higher order diffusion Monte Carlo propagators for linear rotors as diffusion on a sphere: Development and application to $\mathrm{O}_{2} @ \mathrm{He}_{n}$
}

\author{
Massimo Mella a),b) \\ Dipartimento di Scienze Chimiche ed Ambientali, Università degli Studi dell'Insubria, \\ via Lucini 3, 22100 Como, Italy
}

(Received 21 July 2011; accepted 26 August 2011; published online 19 September 2011)

\begin{abstract}
Exploiting the theoretical treatment of particles diffusing on corrugated surfaces and the isomorphism between the "particle on a sphere" and a linear molecule rotation, a new diffusion kernel is introduced to increase the order of diffusion Monte Carlo (DMC) simulations involving linear rotors. Tests carried out on model systems indicate the superior performances of the new rotational diffusion kernel with respect to the simpler alternatives previously employed. In particular, it is evidenced a second order convergence toward exact results with respect to the time step of dynamical correlation functions, a fact that guarantees an identical order for the diffusion part of the DMC projector. The algorithmic advantages afforded by the latter are discussed, especially with respect to the "a posteriori" and "on the fly" extrapolation schemes. As a first application to the new algorithm, the structure and energetics of $\mathrm{O}_{2} @ \mathrm{He}_{n}(n=1-40)$ clusters have been studied. This was done to investigate the possible cause of the quenching of the reaction between $\mathrm{O}_{2}$ and $\mathrm{Mg}$ witnessed upon increasing the size of superfluid $\mathrm{He}$ droplets used as a solvent. With the simulations on $\mathrm{O}_{2}$ indicating a strong localization in the cluster core, the behaviour as a function of $n$ is ascribed to the extremely fluxional comportment of $\mathrm{Mg} @ \mathrm{He}_{n}$, which dwells far from the droplet center, albeit being solvated, when $n$ is large. @ 2011 American Institute of Physics. [doi:10.1063/1.3639190]
\end{abstract}

\section{INTRODUCTION}

With experiments involving superfluid He droplets being extended and perfected as time goes by, more efficient and powerful theoretical methods become necessary to interpret experimental results and solve the scientific puzzles that the latter pose occasionally. To highlight a few examples of the contribution that theory can provide in this context, one may start from the recent work by Hernando et al. ${ }^{1}$ on $\mathrm{Mg}$ and $\mathrm{Mg}_{2}$ in He droplets, where the solubility of the latter species was studied employing a density functional theory (DFT) based approach. The results indicated robust interpretations for the electron-impact ionization experiments carried out in Ref. 2 suggesting a nearly surface-like state for $\mathrm{Mg}$, as well as for the resonant 2-photon-ionization experiments on the droplet doped with more $\mathrm{Mg}$ atoms indicating a "foam-like" state for the latter when dispersed in He. ${ }^{3}$ Similarly, the investigation of the energetics, structure, ${ }^{4}$ and, at least to some extent, dynamics ${ }^{5}$ of $\mathrm{AgHe}_{n}$ with $\mathrm{Ag}$ in the ground and excited state has contributed to clarify some of the intricate details of its post-excitation relaxation. ${ }^{6,7}$

Despite the successes highlighted above, a few scientific riddles still roam the arena occupied by the application of $\mathrm{He}$ droplets. For instance, it is interesting to recall the fact that the excitation spectrum of the lowest triplet state of $\mathrm{Ag}_{2},{ }^{3} \Sigma_{u}$, has recently been recorded regardless of the fact that the solubility of $\mathrm{Ag}$ in $\mathrm{He}_{n}$ is expected to favor the formation of the more stable singlet. ${ }^{8}$ Whether this finding is an indication of

\footnotetext{
a) On leave from School of Chemistry, Cardiff University, Main Building, Park Place, Cardiff CF10 3AT, United Kingdom.

b) Electronic addresses: Massimo.Mella@ uninsubria.it and MellaM@cardiff. ac.uk.
}

a two-step process to form singlet $\mathrm{Ag}_{2}$ via the triplet or simply due to some inefficiency in preferentially populating the lowest energy state is an issue that would deserve an investigation involving a detailed dynamical treatment of the formation process.

To us, the progressive disappearance of the chemiluminescence associated with the reaction between $\mathrm{Mg}$ atoms and oxygen molecules sorbed by helium clusters upon increasing the size of the He nano-cryostat ${ }^{9}$ is even more puzzling. Perhaps naively, one would imagine that dilution due to a large droplet may affect the kinetics of such a simple reaction but not its outcome, which is clearly not the case. It is thus with the goal of addressing this paradox that we therefore set out to investigate properties of $\mathrm{O}_{2} @ \mathrm{He}_{n}$ using atomistic quantum simulations; this is in view of the fact that $\mathrm{Mg} @ \mathrm{He}_{n}$ has already been studied extensively. ${ }^{1,10}$

In respect to the latter goal, we note that, while quantum statistical simulations on atomic clusters have progresses to the point where it is possible to simulate a few hundreds of quantum atoms in a reasonable amount of computer time, thanks to much improved algorithms employing higher order thermal density matrices, ${ }^{11-16}$ similar improvements have been sparse and occasional in the case of systems containing rigid bodies. The latter "state of affair" should be attributed more to the complicated topology of the coordinate space needed to describe both internal and external degrees of freedom (e.g., torsions and overall orientation) than to the lack of cunning from investigators. In fact, a few strategies have recently become available to carry out diffusion Monte Carlo (DMC) (Refs. 17-19) and path integral Monte Carlo (PIMC) (Refs. 20 and 21) in curved manifolds that at least guarantee first order convergence with respect to the time step or the 
number of slices taking into account axel precession and Coriolis couplings that were originally neglected. ${ }^{23,24}$ Building on this advances, a clever implementation of estimators such as for the internal energy allows a third order convergence with respect to the number of PIMC slices. ${ }^{22}$ This notwithstanding, there appears to be no clear way forward for improving DMC projectors and PIMC thermal density matrices beyond first order, a possibility that has already been shown to provide more efficient algorithms despite the higher cost per step. Thus, to make an attempt to fill the methodological gap left in the quantum statistical simulations of rigid molecule aggregates, we endeavored to provide the community with a higher order algorithm for linear molecules, which would suit our interest in simulating linear rotor-atomic aggregates. To do so, it was opted to exploit recent advances in the theory of diffusion on rough/curved surfaces, the application of the latter theories to our context being described in more detail in Sec. II.

The outline of this manuscript is as follows. Section II contains a more complete description of the "state of the art" in quantum stochastic simulations and describes the approach chosen to overcome some of the current limitations. It also presents the results obtained carrying out simulation on two model systems. Section III provides the results of our DMC simulations on $\mathrm{O}_{2} @ \mathrm{He}_{n}$, including also a discussion relevant for the interpretation of the experiments described in Ref. 9. Lastly, Sec. IV gives our final comments on this investigation effort together with further venues of applications of the new algorithm introduced.

\section{THEORY}

Atomic units will be assumed throughout the manuscript unless otherwise stated.

The theory of DMC relies completely on the possibility of either writing a convenient analytical form for the projector $e^{-t \mathcal{H}}$ or developing algorithms that allow it to be sampled with some order of error with respect to time fractionation (i.e., the time step $\delta t$ ). In this respect, the Trotter factorization and the Baker-Cambell-Hausdorf formula are often used to provide the practitioner with high order approximations, which tend to reduce the systematic errors in computed observables for systems that are simulated in the $3 \mathrm{~N}$ Cartesian space needed to describe the position of point-like particles. Noteworthy, one has always a way to decompose $e^{-t \mathcal{H}}$ to second order in $\delta t$ as a product of operators in the $3 \mathrm{~N}$ Cartesian space whose action can be exactly simulated in the latter situations.

When the same approach needs to be applied to the curved manifold such as the one that is required in the case of systems containing rigid bodies or, more generally, subjected to holonomic constraints, one quickly encounters an additional problem induced by the fact that a closed form of the action of the kinetic energy operators describing rotation and torsion in the system is not readily available. Due to this, several simple and convenient approximations have been used to circumvent such problem, with a common trait, however to be at best first order with respect to $\delta t$. Among the diffusional evolution operators which can at most provide linear convergence with respect to $\delta t$, we recall the approach taken by Buch $^{23}$ and formalized by Viel et al., ${ }^{25}$ as well as the more modern approach based on stereographic projections and DeWitt formulae introduced by Aviles and Curotto ${ }^{17}$ and recently brought to higher levels of efficiency by Luan, Curotto, and Mella. ${ }^{26}$ These choices, albeit convenient for practical purposes, have the drawback of degrading the overall order of the DMC algorithms preventing the use of long time steps and the exploitation of many of the most recent extrapolation approaches for improving efficiency.

In this section, we wish to describe a simple but convenient approach that allows one to simulate the action of the rotational kinetic energy operator $\Lambda^{2} / 2 I$ (or better of its diffusional projector $e^{-t\left(\Lambda^{2} / 2 I\right)}$ ) for a linear molecule whose error term $O\left(\delta t^{n}\right)$ has $n=1,2$, or 3 . This is based on the work by Faraudo, ${ }^{27}$ who developed analytical approximations for the probability distribution function of the diffusion length along a 2D surface expressed in geodesic coordinates $(u, v)$ (i.e., the generalized latitude and longitude) and the isomorphism between the imaginary-time Schroedinger equation for the free rotation of a linear molecule with inertia moment $I$ (or a point particle of mass $m$ on a sphere with square radius $I / m$ ) and the diffusion equation of a species with a diffusion coefficient $D=1 /(2 I)$ on a unit sphere. Equation (51) in Ref. 27 provides the much needed expression, developed by means of perturbation theory for an isotropic surface (i.e., one for which the metric tensor $G(u, v)$ of the manifold defined by the surface depends only on the geodesic distance $u$ ), in terms of the Gaussian curvature $\mathcal{K}_{0}$ at the location of the diffuser and some related quantities, namely, a characteristic time $\tau_{0}^{-1} \equiv 4 D\left|\mathcal{K}_{0}\right|$ and radius $R_{0} \equiv\left|\mathcal{K}_{0}\right|^{-1 / 2}$. In the case of diffusion over a sphere of radius $R$, for which the curvature is constant in every point, the mentioned equation simplifies remarkably and it can be written as

$$
\begin{aligned}
P(u, t)= & \frac{e^{-u^{2} /(4 D t)}}{4 \pi D t}\left[1+\frac{1}{12}\left(\frac{u}{R}\right)^{2}+\frac{1}{12} \frac{t}{\tau_{0}}\right. \\
& \left.+\frac{1}{240}\left(\frac{t}{\tau_{0}}\right)^{2}+\cdots\right]
\end{aligned}
$$

with $u$ being the length of the arc diffused after time $t$. The terms omitted in the series in Eq. (1) are of order four or above with respect to $u / R$ and three for $t / \tau_{0}$. In the case of a sphere, $u$ is clearly measured along one of the great circles passing through the starting point of the diffusion, and it is thus easy to derive an algorithm that can be used to sample the diffusion of the sphere itself. To do so, we recall that Eq. (1) must be multiplied by $\sqrt{G(u)} d u=R \sin (u / R) d u$ to generate the probability of taking a step of length $u$ after time $t$, so that we ought to sample the form in Eq. (2) where we set $\mathcal{A}(t) \equiv 1+(1 / 12)\left(t / \tau_{0}\right)+(1 / 240)\left(t / \tau_{0}\right)^{2}:$

$$
\begin{aligned}
P(u, t) \sqrt{G(u)} d u & =\frac{e^{-u^{2} /(4 D t)}}{4 \pi D t}\left[\mathcal{A}(t)+\frac{1}{12}\left(\frac{u}{R}\right)^{2}\right] R \sin (u / R) d u \\
& \leq \frac{e^{-u^{2} /(4 D t)}}{4 \pi D t}[\mathcal{A}(t)+1] R \sin (u / R) d u \\
& \leq \frac{e^{-u^{2} /(4 D t)}}{4 \pi D t}[\mathcal{A}(t)+1] u d u
\end{aligned}
$$


The two inequalities descend from the fact that $(1 / 12)(u / R)^{2} \leq 1$ for $0 \leq u \leq \pi R$ and it is always $R \sin (u / R) \leq u$ over the same interval. The last probability can be easily sampled employing the inversion method since the cumulative probability $\int_{0}^{\pi R}\left(e^{-u^{2} /(4 D t)}\right) /(4 \pi D t) u d u$ can be easily obtained analytically. In turn, this allows the correct probability density for $u$ to be sampled using a rejection method. A summary of the algorithm implemented to simulate the rotational diffusion on a sphere is provided in Appendix IV.

Different from what it is usually done when testing a new projector (such as new integrators for the Langevin equation $^{12}$ ) in Cartesian space, in this case we cannot rely on the fact that the sampled limiting distribution for $t \rightarrow \infty$ should have an error proportional to some power of the time step. In fact, it is trivial to demonstrate that any form for $P(u, t)$ that is even in $u$ would, in the long run, generate an uniform distribution of points on the sphere. Conversely, what we ought to test is the accuracy of the relaxation dynamics itself, which could be gauged comparing the exact (or extremely accurate) and simulated values of an observable that is capable of tracking the dynamics as a function of the time step chosen. In this respect, Fig. 1 shows $\langle\cos [\beta(\delta t)]\rangle$ for three different approximations derived from Eq. (2), namely, one for which $\mathcal{A}(t) \equiv 1$ and $(1 / 12)(u / R)^{2}$ are omitted, one for which the latter term is retained but it is still $\mathcal{A}(t) \equiv 1$, and a last one for which all terms in Eq. (1) are retained. Here, $\cos (\beta)$ is the $\operatorname{cosine}$ of the angle diffused after the time $t=(10 D)^{-1}$ when $D=10^{-5}$ a.u. as a function of time step $\delta t$. Notice that we have not included in the comparison provided by Fig. 1 the approaches suggested in Refs. 17,23, and 25 due to the fact that we expect a performance similar or worse to the one provided by the simplest among our approximations.

The value of $t$ was chosen in order for the expectation value to be representative of a diffusion that is highly unlikely to reach a point opposite to the starting location, helping us to

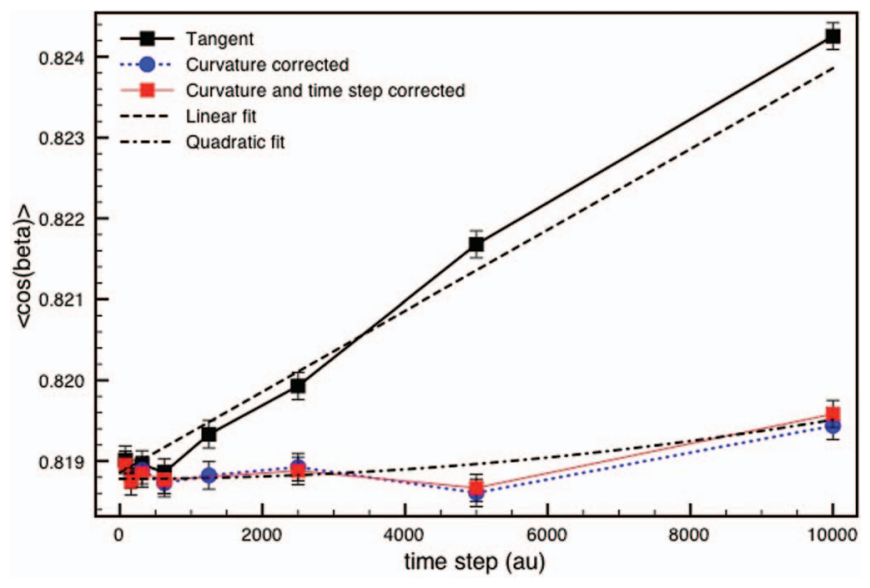

FIG. 1. Convergence of $\langle\cos [\beta(\delta t)]\rangle$, the angle diffused after a time $t=(10 D)^{-1}$, as obtained simulating diffusion with three approximations derived from Eq. (1). "Tangent" represents data obtained with no correction to the basic algorithm, while "curvature" and "curvature and time step" corrected results are from algorithms employing, respectively, $\mathcal{A}(t)$ $\equiv 1$ and $\mathcal{A}(t) \equiv 1+(1 / 12)\left(t / \tau_{0}\right)+(1 / 240)\left(t / \tau_{0}\right)^{2}$ with the distance dependent correction $u^{2} /\left(12 R^{2}\right)$. verify the correctness of the short time dynamics. The starting distribution of points was sampled uniformly generating points in the unit cube whose center coincides with the sphere one and rescaling their vector length to unit module. In this way, we concentrate more points around the intersections between the sphere surface and the diagonal of the cube itself with the advantage that the non-uniform initial distribution allows one a more insightful test of the diffusion algorithms. Notice that a value for $D$ such as the one employed in these simulations is relevant for the rotational diffusion of first row diatomic molecules, such as $\mathrm{N}_{2}$ and $\mathrm{O}_{2}$.

As one can easily notice, the basic approximation to surface diffusion $(\mathcal{A}(t) \equiv 1$ and no $u$-term) provides one with a robust linear convergence toward the exact diffused angle; keeping in mind that this approach is at least equivalent (if not better due to the use of the exact volume element $R \sin (u / R)$ instead of $u$ ) than what was often used to simulate a random walk on corrugated surfaces, ${ }^{28}$ this finding is not surprising under the light of the error analysis carried out in Ref. 28. Upon introducing the $u$-dependent term alone, one notices a marked improvement so much that it is difficult to understand whether the convergence order with respect to the time step is linear, as expected due to the lack of the $t$-dependent terms, or supralinear. In this respect, notice that $(1 / 12)(u / R)^{2}$ $\sim 0.067$ (as estimated from $\left\langle u^{2}\right\rangle$ in our simulations) and that $(1 / 12)\left(t / \tau_{0}\right) \sim 0.033$ for the longest of the time step shown in Fig. 1. However, the effect of the coordinate-dependent term is more substantial than that predicted by these simple estimates, since it pushes the distribution toward larger $u$, while the time-dependent term has the net effect of slightly modulating this "push." As a consequence of this reasoning, one should also expect a limited improvement upon increasing the order of the time-dependent correction introduced, a conclusion that is readily verified by our numerical results.

While the latter conclusion seems to suggest that only a limited absolute improvement may come from using high order approximations in $\delta t$ to describe rotational diffusion on a sphere in DMC simulations once the $(1 / 12)(u / R)^{2}$ term is introduced, the assurance of a well defined order provides one with the chance of robustly extrapolating the simulation results, whose main systematic error is expected to derive from the non-commuting nature of the kinetic and potential energy operators. Thus, the theory put on solid basis in Ref. 12 for a posteriori extrapolation ${ }^{29}$ allows one to reduce substantially the systematic errors associated with the commonly employed symmetric Trotter splitting even when simulating linear rigid bodies. In this respect, we would like to remark that the error reduction witnessed in Fig. 1 covers the whole range of time steps commonly used to simulate He droplets ( $\delta t \leq 600$ a.u.) or Ne containing species $\left(\delta t \leq 2000\right.$ a.u.). ${ }^{13}$

To put the above ideas under test, we decided to simulate a model system employing DMC with the same approximations to the diffusion kernel used to study the convergence of $\langle\cos (\beta)\rangle(\delta t)$. The system is composed of a single dipole interacting with an uniform electric field oriented along the z-axis and restrained to vibrate around the origin of a set of Cartesian axis by a harmonic potential acting on the dipole center of mass. This model is a transposition to a more complex 


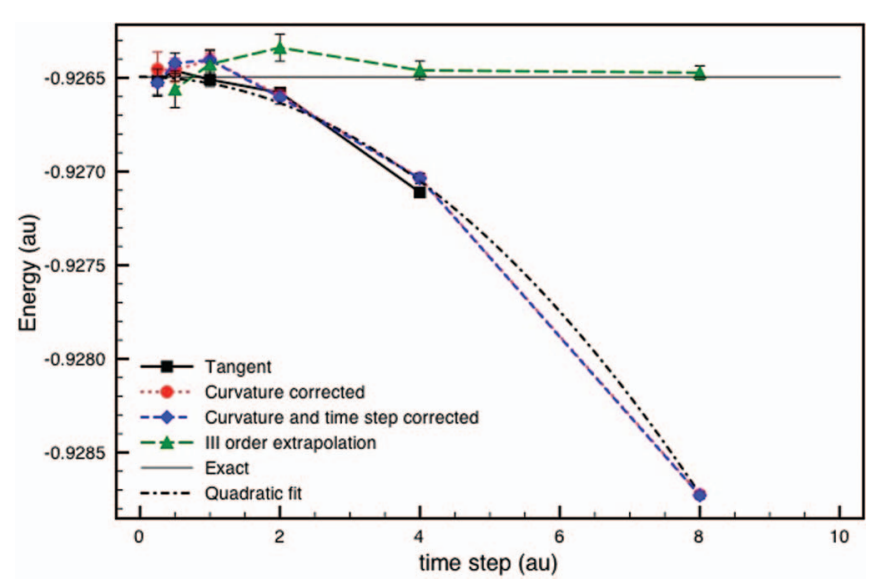

FIG. 2. Convergence of $\langle V\rangle(\delta t) \equiv E_{0}$ as obtained using no-IS DMC simulations for the rigid dipole in the harmonic well described in the text. "Tangent" represents data obtained with no correction to the basic algorithm, while "curvature" and "curvature and time step" corrected results are from the algorithm employing, respectively, $\mathcal{A}(t) \equiv 1$ and $\mathcal{A}(t) \equiv 1+(1 / 12)\left(t / \tau_{0}\right)$ $+(1 / 240)\left(t / \tau_{0}\right)^{2}$ with the distance dependent correction $u^{2} /\left(12 R^{2}\right)$. The agreement between the third order extrapolated energy data and the exact value remarks the robust second order behaviour of the curvature and time step corrected diffusion kernel.

configuration space of the free dipole interacting with an uniform field used by Curotto to demonstrate finite differences approaches in curved manifolds. ${ }^{30}$ The dipole was considered composed of two point-like particles with mass of 414 a.u. each at a distance of 1 bohr; the spring constant for the harmonic well was chosen as $10^{-2}$ hartree/bohr, ${ }^{2}$ while a field of 1 a.u. was employed to parallel the model by Curotto. ${ }^{30}$ The exact energy for this system is -0.926496 hartree. Figure 2 presents the data from our simulations run using a DMC algorithm without importance sampling, its striking feature being the very good agreement in terms of the system's total energy between all levels of approximation employed for the diffusion kernel. This finding, obviously, supports the expectation discussed previously while commenting the results on $\langle\cos [\beta(\delta t)]\rangle$, both with respect to the time step dependency (the most accurate kernel demonstrates a robust second order behaviour deriving from the symmetric splitting $\exp \left[-\delta t V^{\prime} / 2\right] \exp [-\delta t T] \exp [-\delta t V / 2]$ of the thermal density matrix) and of the possibility of a solid a posteriori third order extrapolation. In fact, third order results are all within two standard errors from the exact energy.

\section{DMC SIMULATIONS OF $\mathrm{O}_{2} @ \mathrm{He}_{n}$ CLUSTERS $(n=1-40)$}

DMC simulations on $\mathrm{O}_{2} @ \mathrm{He}_{n}$ clusters have been carried out employing a standard second order symmetric (also known as Trotter) factorization of the projector operator, which differs with the one employed in Ref. 11 only due to the presence of the rotational diffusion part discussed in Sec. II. Worth stressing, it is the fact that the rotational kinetic energy operator in spherical coordinates defined with respect to a laboratory fixed frame commutes with the center of mass Cartesian coordinates, so that the order between rotational and centre of mass diffusions is inconsequential. Importance sam- pling (IS) was introduced by re-weighting the DMC configurations (or walkers) using the ratio between the values of the trail wave function $\Psi_{T}$ before and after the diffusion step. ${ }^{31}$ $\Psi_{T}$ was chosen of the same analytical form previously used to describe $\mathrm{Ag}$ and $\mathrm{Cu}$ in $\mathrm{He}$ droplets $^{32}$ employing $\mathrm{O}_{2}$ center of mass (c.m.)-He atom distances instead of $\mathrm{O}$ atom-He atom distances. This choice serves two purposes, namely, the use of a very efficient wave function that is capable of describing also second shell He atoms if needed and the absence of an angular bias for the $\mathrm{O}_{2} \mathrm{He}$ density. In other words, we allowed DMC to suggest the most appropriate angular distribution between a $\mathrm{He}$ atom and the $\mathrm{O}_{2}$ bond axis.

As usual, we wrote the total interaction potential as a sum of two-body terms; this is justified by the very low polarizability of He atoms and the lack of strong multipole moments in $\mathrm{O}_{2}$. To compare with previous work of ours, we opted for employing the Toennies-Tang-Yang (TTY) form ${ }^{33}$ as He-He pair potential. The 3D potential developed by Groenenboom and Struniewicz (GS) (Ref. 34) was instead used for the $\mathrm{O}_{2}-$ He interaction keeping $\mathrm{O}_{2}$ frozen at its equilibrium distance in vacuum, 2.282 bohr. From the markedly linear dependency of the GS potential on the $\mathrm{O}_{2}$ stretching coordinate around the minimum, we expect our choice of freezing the molecular bond at its minimum to be inconsequential when compared to using the vibrational averaged distance.

The GS potential features two minima, one linear (116.7 $\mu$ hartree at a distance of $6.9 \mathrm{bohr}$ ) and one T-shaped (127.1 $\mu$ hartree, 6.9 bohr), with the T-shaped one being the lowest of the two. The two minima are separated by a barrier that is located $36.7 \mu$ hartree above the lowest stationary point. Compared with the TTY well depth of roughly $33 \mu$ hartree at the He-He distance of $5.6 \mathrm{bohr}$, these energy values suggest as likely that $\mathrm{O}_{2}$ is indeed soluble in helium droplets; it certainly will with classical simulations. However, one ought not to discard the possibility that dynamical many-body effects may modulate somewhat this static picture, as already evidenced previously. ${ }^{35}$ In particular, we aim to establish if helium would preferentially be located in the T-shaped global minima due to the lower potential energy or may indeed delocalize due to quantum effects. Besides, the height of the local He density maximum expected in the first solvation shell around $\mathrm{O}_{2}$, as well as the fluctuation of the latter with respect to the centre of the He droplet, may have bearings on the possible quenching of the $\mathrm{Mg}-\mathrm{O}_{2}$ reaction as discussed in the case of the formation of $\mathrm{Mg}_{n}$ clusters. $^{3}$

With the potentials discussed above, simulations were carried out using 1000 walkers as target population; initial tests deemed such population size to be suitable for our goal of estimating energy differences and investigating solvation details, as well as to provide a population bias of roughly a part per ten thousands (i.e., similar or smaller than the associated standard error) even for simulations not employing the IS procedure (no-IS) to minimize the errors. The latter were used to assess the degree of He delocalization in clusters with $n \leq 8$ exploiting the deep well in the $\mathrm{O}_{2}-\mathrm{He}$ potential to keep them bound. For larger $n$, we were forced to use IS. Similarly, we opted for a time step of 100 a.u. for the no-IS simulations and of 500 a.u. for the IS ones; both values guarantee a bias of the same order of what just discussed. Independent 
TABLE I. Total energy and $\mathrm{He}$ evaporation energy $\Delta_{0}(n)$ $=(E(n)-E(m)) /(n-m)$ for $\mathrm{O}_{2} @ \mathrm{He}_{n}$. The standard error of mean values is roughly one unit of the last decimal digit shown. $m$ is the number of $\mathrm{He}$ atoms in the aggregate closer in size to $\mathrm{O}_{2} @ \mathrm{He}_{n}$ and with $m<n$.

\begin{tabular}{llc}
\hline \hline$n$ & $\mathrm{E}(n)$ (hartree) & $\Delta_{0}(n)\left(\mathrm{cm}^{-1}\right)$ \\
\hline 1 & -0.0000351 & \\
2 & -0.0000714 & -7.95 \\
4 & -0.0001469 & -8.27 \\
6 & -0.000223 & -8.38 \\
8 & -0.000297 & -8.0 \\
10 & -0.000355 & -6.4 \\
14 & -0.000456 & -5.5 \\
18 & -0.000521 & -3.6 \\
20 & -0.000551 & -3.3 \\
25 & -0.000623 & -3.2 \\
30 & -0.000695 & -3.2 \\
40 & -0.000839 & -3.1 \\
\hline \hline
\end{tabular}

of the presence or not of the IS procedure, all simulations were run generating, at least, 250000 new populations, each containing roughly 1000 walkers, a number that was progressively increased upon increasing the system size.

Table I and Fig. 3 provide the results for the total energy and He evaporation energy obtained with DMC simulations as a function of the number of He atoms, $n$. The evaporation energy at each $n$ is defined as the incremental ratio (i.e., change per atom) of a cluster energy with respect to the closer species that is smaller in size. As usual, this quantity allows one to extract a wealth of information about the cluster structure. In fact, comparing with previous results, ${ }^{4,32,36}$ it clearly emerges that a compact first solvation shell is formed around the oxygen molecule and it may be closed already at $n=18$. We also found an excellent agreement between the evaporation energy in the range $20 \leq n \leq 40$ for our clusters and large He clusters containing strongly binding species, ${ }^{37,38}$ which we interpret as a strong suggestion for the presence of a second solvation shell in the size range $20 \leq n \leq 40$. In other words, we consider that the almost identical evaporation energy shown by large clusters containing different impurities is, in practice, indicating the presence of similar environments due to the shielding of the He-impurity interaction at long distances. Finally, it is also clearly evident the presence of a stabilizing dynamical many-body effect for $n \leq 6$, which is washed out by the excluded volume effect (i.e., higher kinetic energy due to less room available per atom) for larger $n$.

A more precise characterization of the structural features of doped He droplets is usually carried out by presenting various probability distribution functions. In our case, this is done in Figs. 4 and 5, which, respectively, show the 2D (in radial and angular Jacobi coordinates) probability distribution of $\mathrm{He}$ atoms around the oxygen molecule for a few selected clusters ( $n=2,8$, and 40) and the distribution of oxygen molecule c.m. with respect to the centroid of the He moiety. All pair distributions were obtained averaging over irrelevant coordinates either $\Psi_{0}$ or $\Psi_{0} \Psi_{T}$ as sampled using, respectively, DMC simulations without or with IS. To provide a few additional visual particulars, we also show a typically sampled structure for $n=2,8,18$, and 40 in Fig. 6 .

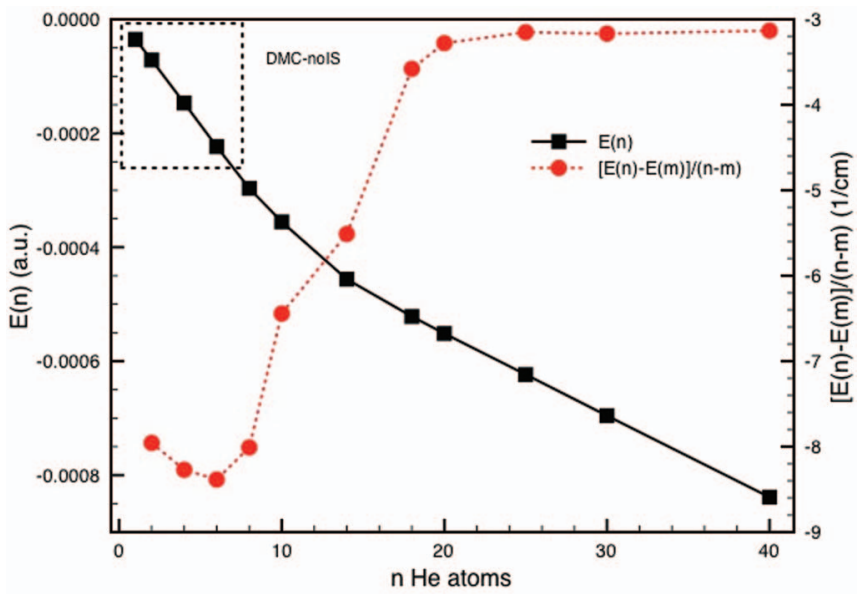

FIG. 3. Total (square, left axis, atomic units) and evaporation energies (circle, right axis, $\mathrm{cm}^{-1}$ ) for $\mathrm{O}_{2} @ \mathrm{He}_{n}$ as a function of the number of $\mathrm{He}$ atoms, $n . m$ is the number of $\mathrm{He}$ atoms in the aggregate closer in size to $\mathrm{O}_{2} @ \mathrm{He}_{n}$ and with $m<n$. The data for the size range $1 \leq n \leq 8$ were obtained using no-IS DMC simulations; larger clusters have been studied employing IS DMC to reduce statistical errors and to prevent second shell $\mathrm{He}$ atoms evaporation. Standard errors are of comparable size or smaller than symbols.

From Fig. 4, it is clear that He is completely delocalized around the molecule; nevertheless, two smooth maxima are found in the linear and T-shape configurations for atoms in the first solvation shell. Such maxima are present in all the clusters we studied and are thus largely independent of the cluster size. Their height, however, increases upon increasing the number of $\mathrm{He}$ atoms in the aggregate as commonly found for solvated impurities, indicating the presence of a "pressure" effect due to the second solvation shell atoms (see Fig. 5). Worth noticing, it is the fact that the local density in the first shell is close to the bulk He density already at $n$ $=8$, i.e., somewhat before the shell closure. This outcome is clearly related to the well depth of the interaction between $\mathrm{He}$ and $\mathrm{O}_{2}$, as previously evidenced by the calculation on strongly binding species. In fact, to find a similar density in the case of $\mathrm{Ag}$ (considered a strong binding atomic dopant), one has to reach $n \sim 20,{ }^{32}$ while a density of 0.0045 a.u. (i.e., as $\mathrm{O}_{2} \mathrm{He}_{40}$ ) is seen only in $\mathrm{AgHe}_{90}$. Besides, the small outward shift of the first solvation shell peak seen in the He density upon increasing $n$ is partially due to the improved sphericity of the He solvation layer.

Figure 5 also completely ascertains that $\mathrm{O}_{2}$ is fully solvated at any value of $n$ by giving evidence that its c.m. always lies close to the c.m. of the He atoms. In this respect, we notice that the fluctuation allowed to the oxygen molecule with respect to the He cluster centroid is very small (roughly 2 bohr for the largest cluster treated), somewhat smaller than that for $\mathrm{Ag}$ that is already a strongly binding neutral species. ${ }^{32}$ Indications supporting our general interpretation for the structure of $\mathrm{O}_{2} @ \mathrm{He}_{n}$ clusters can also be obtained by other angular and spatial distributions or by the direct visualization of atomic positions represented by the DMC walkers (see Fig. 6); however, we refrain from presenting the former for the sake of space and simply indicate that, for clusters with $2 \leq n \leq 12$, He atoms tend to aggregate together preventing the filling of 


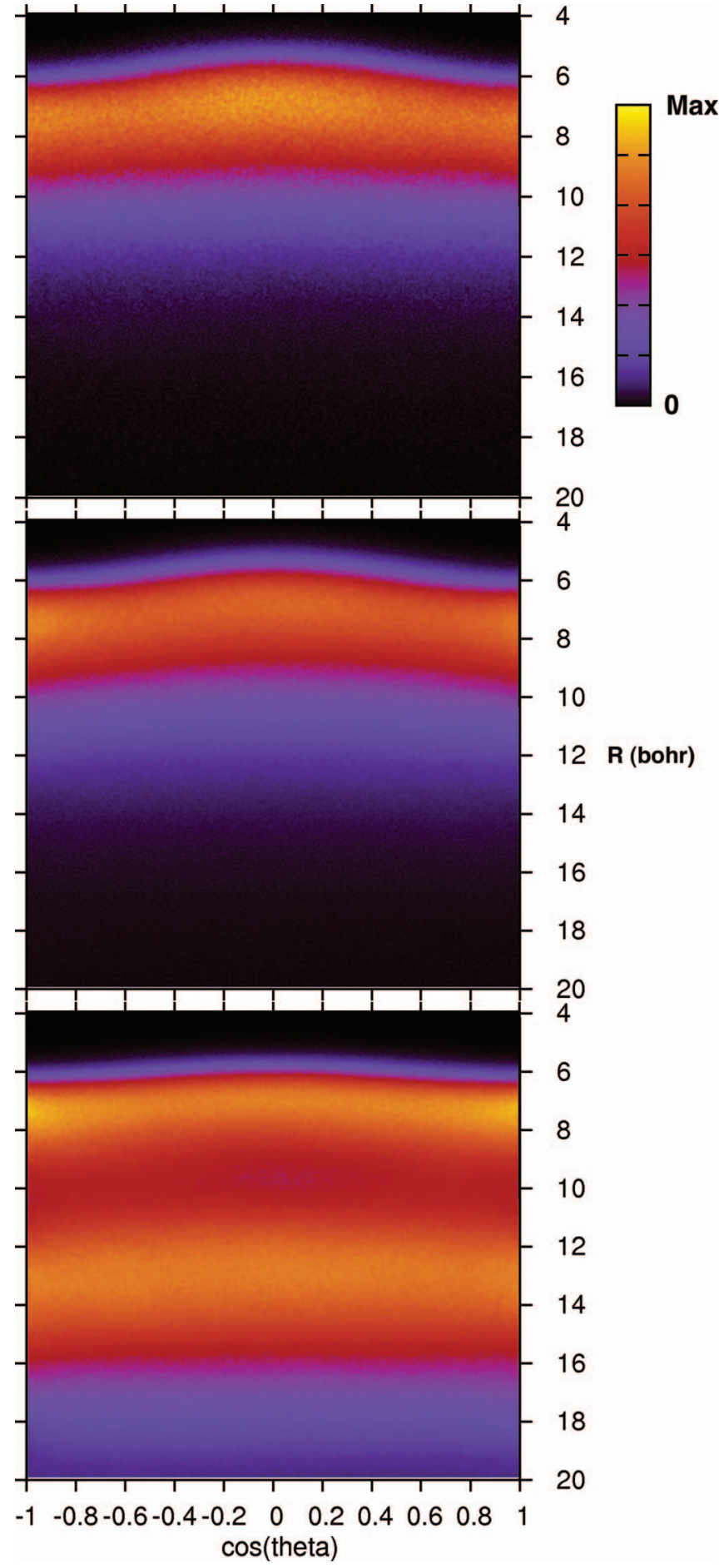

FIG. 4. Two-dimensional relative He density. Coordinates are the distance from the $\mathrm{O}_{2}$ center of mass and the cosine of the angle formed with the molecular axis. Colors indicate the relative values with respect to the maximum density for $n=2$ (top), 8 (middle), and 40 (bottom).

the expected toroidal belt due to the T-shaped global minimum in the GS potential.

\section{DISCUSSION AND CONCLUSIONS}

In this work, we have studied the energetics and structural details of $\mathrm{O}_{2} @ \mathrm{He}_{n}$ clusters $(n=1-40)$ employing state of the art interaction potentials and quantum statistical simulation

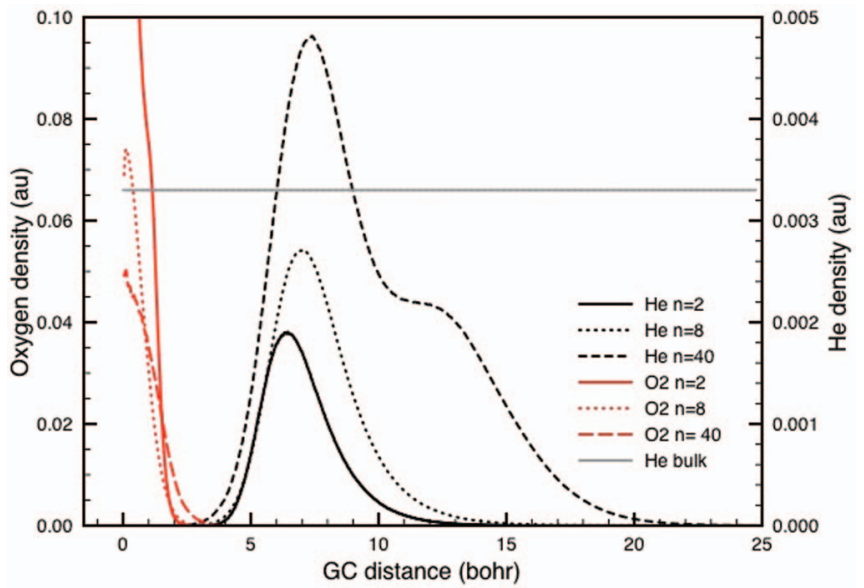

FIG. 5. He (black lines, right axis) and $\mathrm{O}_{2}$ (red lines, left axis) probability distribution functions with respect to the distance from the geometrical centre of the He moiety. The horizontal line represents the bulk He density in similar thermodynamical conditions. The cluster sizes shown in the figure have been chosen as representative cases for the three regimes of shell filling discussed in the main text. Oxygen densities are normalized to unit value, while $\mathrm{He}$ densities are normalized to the atom number $n$.

methods. In fact, we developed a new DMC algorithm for linear rigid rotors that affords a higher order of convergence with respect to the time step employed during a simulation and consequently a smaller bias than previously used schemes when it come to evaluate correlation functions. Such improvements come at the cost of a limited increase in the complexity of the algorithm and with no additional computational time spent per DMC step, thanks to an efficient implementation of

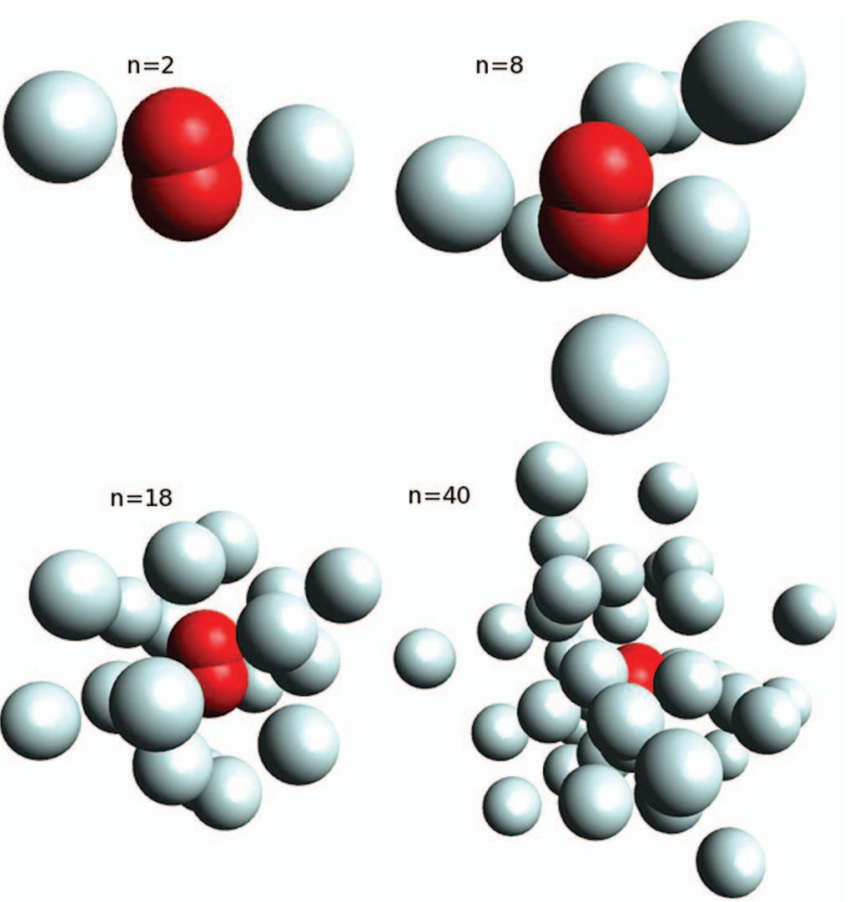

FIG. 6. Typically sampled structures for $n=2,8,18$, and 40 . Notice the tendency of $\mathrm{He}$ atoms to aggregate together evident in $\mathrm{O}_{2} \mathrm{He}_{n}$. This seems to slow down the completion of the "belt" that ought to be formed due to the T-shaped minimum in the interaction potential and favors the occupation of the linear minima. 
the sampling of the rotational displacement along a great circle. Benefits from the improved algorithm discussed in this paper could be harvested by methods, such as reptation quantum Monte Carlo (RQMC) (Ref. 39) and PIMC, both of which can be used to compute imaginary time rotational correlation functions to extract the change in rotational constants due to the solvating environment. ${ }^{40-46}$ In this respect, RQMC would necessitate of a somewhat new implementation introducing a robust second order approximation to the Langevin (drift and diffusion) kernel before it could collect the advantages provided by using Eq. (1), while one would simply require to modify the acceptance/rejection step in PIMC.

As far as statistical expectation values are concerned, however, the results presented indicate that only very limited improvements are to be expected from using a higher order approximation of the diffusion kernel for a spherical surface manifold. Nevertheless, the third order short time error of the most accurate approximation tested in this work paves the way toward the use of more sophisticated approaches based on the theory of stochastic differential equations. In fact, the possibility of a posteriori extrapolating the data to reduce the time step bias relies on the same sufficient conditions ${ }^{12}$ that allow one to extrapolate "on the fly."13 Albeit not attempted in this work due to the small system sizes with respect to today's computer capabilities, it is expected that implementing on the fly the time step extrapolation would give an efficiency boost similar to what obtained for atomic aggregates with no additional computational overheads.

As discussed in the Introduction, the main thrust for the presented work was the disappearance of the chemiluminescence associated with the reaction between a few $\mathrm{Mg}$ atoms and $\mathrm{O}_{2}$ (Ref. 9) upon increasing the He droplet size. Whereas both an increase of droplet radius and of the $\mathrm{Mg}$ background pressure should favor the (early) pick up of more $\mathrm{Mg}$ atoms per cluster and the (later) capture of $\mathrm{O}_{2}$ due to volume effects, the experiment indicated that chemiluminescence decreased in the first case. Whether such an effect was due to solubility issues or simply due to a reduced number of He droplets in the beam could not be clarified at that time. In this respect, the data obtained with our simulations make clear that the oxygen molecule is highly soluble in He droplets and it should be expected to dwell very close to the droplet c.m. and to afford only limited (Gaussian) fluctuations from that dynamical equilibrium position. Consequently, $\mathrm{O}_{2}$ should be expected to sink into He droplets as soon as the impact energy is dispersed by the quantum environment. Conversely, DFT results, ${ }^{1}$ and to some extent also DMC ones, ${ }^{10}$ suggest a different behaviour for $\mathrm{Mg}$ atoms in the same environment, with $\mathrm{Mg}$ atoms being delocalized inside the droplet, while being restrained from visiting frequently (or at all, vide infra) the He aggregate centroid. In fact, such limitation becomes progressively more evident and stringent comparing the $\mathrm{Mg}$ distribution functions obtained by Hernando et al. for $n=1000$, 2000, and 10000 (see Fig. 6 in Ref. 1, where it becomes explicit noticing that a droplet containing $10000 \mathrm{He}$ atoms should have a radius roughly twice as large as the one with 1000 heliums). Taken together, the comportment extracted for $\mathrm{Mg}$ and the oxygen molecule from theoretical calculations pave the way for a rationalization of the dependency of the chemiluminescence on the droplet size. In fact, for a given number of $\mathrm{Mg}$ atoms picked up before the oxygen molecule, the probability of coming close to a sinking oxygen molecule decreases upon increasing the droplet size $n$ roughly as $n^{-2 / 3}$. This is under the assumption that the $\mathrm{Mg}$ atoms are expected to remain "dispersed" and not to condense into $\mathrm{Mg}$ clusters. ${ }^{3}$ It thus becomes more likely for $\mathrm{O}_{2}$ to pass the range of distances from the droplet c.m. where $\mathrm{Mg}$ atoms dwell without interaction with the latter upon increasing the droplet radius. Once this has happened, the limited penetration of $\mathrm{Mg}$ into the droplet and the strong localization of $\mathrm{O}_{2}$ close to the center of the latter make the probability for the reactants to come in contact and generate hot products capable of emitting infrared light very low. Noteworthy, the mechanism just exposed is also in agreement with the fact that in the presence of $\mathrm{Xe}$ clusters products are generated and detected in the mass spectrometer in substantially larger abundance than without, but no chemiluminescence was detected. In fact, we would expect Xe clusters, added before both $\mathrm{Mg}$ and $\mathrm{O}_{2}$, either to "nucleate" and sink Mg clusters as done with Ba (Ref. 47) or to act as an attractor close to the droplet center, thanks to their larger volume than $\mathrm{O}_{2}$. In either cases, the reaction would become more likely and allowed to take place deeper inside the He aggregate, thus facilitating the dissipation of the fraction of the reaction energy which is converted into the product translational energy. In the same way, we are in the position of suggesting a possible explanation for the interesting observations made in Ref. 9 that single $\mathrm{Mg}$ atom doped droplets do not produce chemiluminescence and are likely to eject $\mathrm{MgO}_{2}$ or other products before the reaction energy is dissipated. From our and Hernando et al. results, ${ }^{1}$ it becomes clear that $\mathrm{Mg}$ and the oxygen molecule are likely to "collide," following the pick up of the latter species and only while it is sinking toward the aggregate center, i.e., just under the droplet surface, a situation that appears likely to favor a prompt ejection of the energized product, thanks to a conversion of internal energy into center of mass energy and leading to the absence of the product signal in the mass spectrometer.

While the juxtaposition of DMC and DFT results allows one to discuss a possible mechanism explaining the chemiluminescence and mass spectrometry results, it does not provide any rationale for the surprisingly high value $\left(5 \times 10^{4} \mathrm{~s}^{-1}\right)$ of the first order reaction constant estimated by Krasnokutski and Huisken ${ }^{9}$ (see Ref. 48 for a comparison with gas phase data). At the moment, it seems difficult to disentangle possible contributions to this high value due to the diffusion of $\mathrm{Mg}$, the possibility of a strong interaction between $\mathrm{Mg}$ and triplet $\mathrm{O}_{2}$, and the effect of the quantum solvent on shielding the dopants from reacting or favoring possible charge exchange/nonadiabatic transitions between different potential energy surfaces due to the lack of accurate quantitative data. In this respect, it should be feasible to generate adiabatic surfaces involving $\mathrm{MgO}_{2}$ to clarify better the energetics involved (e.g., the presence and height of the suggested barriers and the effect of He on the energy of possible products) as well as to study (e.g., with DFT, which allows to study large droplets) the effects due to the quantum solvent when both reactants are doping a droplet. At the moment, we are carrying out a preliminary investigation on the energetics 
involved in the reaction itself, which was previously suggested to proceed by means of a mechanism unlike the classical "harpoon mechanism." 48

In conclusion, we would like to highlight further avenues of methodological exploration that this work has opened. We begin noticing that it is possible to decompose the rotational diffusion of a symmetric rotor (e.g., water and ammonia) in two contribution, namely, the precession of its principal axis and the rotation around it. Clearly, the two operations do not commute, but a symmetric splitting similar to what was commonly done to separate diffusion and branching in DMC would provide a second order algorithm that could be implemented exploiting the approach discussed in this work. We expect this to be immediately feasible for DMC simulations, while it may require a few adjustments when it comes to the implementation in PIMC codes. The advantage provided by this methodological step would be harvested for large systems and in conjunction with an on the fly extrapolation, paving the way to study the ground state and thermal density of molecular quantum systems at a reduced cost. Besides, the implementation of a robust second order algorithm describing the diffusion on the torsional $\mathcal{S}^{1}$ manifold needed for what just discussed would have also an impact in the quantum thermal simulations of systems with rotational bonds such as polymers.

\section{ACKNOWLEDGMENTS}

The author would like to acknowledge useful discussions and comments by Gabriele Morosi and Dario Bressanini, as well as suggestions received by Emanuele Curotto and Nadine Halberstadt. The author also acknowledges the financial support provided by the "Rientro dei Cervelli" scheme funded by the italian Ministry for the University and Research (MIUR).

\section{APPENDIX: SUMMARY OF THE ALGORITHM FOR THE DIFFUSION ON A SPHERE}

This appendix provides a summary of the algorithm implemented to sample the diffusion of a point particle with diffusion coefficient $D=(2 I)^{-1}$ on an unit sphere. For convenience, the position of the particle on the sphere is described by the Cartesian coordinates of a unit vector $(\mathbf{v} \equiv(x, y, z)$ with $\left.x^{2}+y^{2}+z^{2}=1\right)$ despite the fact that only two scalar (e.g., $\phi$ and $\theta$ in spherical coordinates) would suffice. For each rotor in the simulated system, the vector $\mathbf{v}$ would be the part of the set of coordinates defining a DMC configuration (or walker) indicating the spatial orientation of the linear rotor. Atomic particles and rotor c.m.'s would be represented as usual by three Cartesian coordinates.

(1) Define the value of $\mathcal{A}(t)$ on the basis of the order of the algorithm chosen according to Eq. (2);

(2) Compute $v_{\max } \equiv \int_{0}^{\pi R}\left(e^{-u^{2} /(4 D t)}\right) /(4 \pi D t) u d u$, the cumulative probability of displacing the position on the sphere anywhere in the range of $0 \leq u \leq \pi R$;

(3) Choose the displacement $u$ along a great circle randomly as $u^{2}=-\ln \left[1-\phi_{u}\right]\left(\tau / \tau_{0}\right)$, where $\phi_{u}=\xi \times v_{\max }$ and $\xi$ is a uniform random variate with $0 \leq \xi \leq 1$;
(4) Either accept the displacement $u$ with probability $p_{a c c}$ $=\left(\mathcal{A}(t)+u^{2} / 12\right) /(\mathcal{A}(t)+1)$ or go back to step 3 ;

(5) Compute $\mathbf{v}_{u}=\mathbf{v} \cos (u)$, which lies along $\mathbf{v}$ but it has a length so that the intersection of the perpendicular plane to it on which its terminal point lies defines all possible landing positions for a step of length $u$ on the unit sphere;

(6) Generate a normalized vector $\mathbf{a}$ orthogonal to $\mathbf{v}_{u}$ from the Cartesian versor with the lowest overlap (e.g., by subtracting the projection of $\mathbf{v}_{u}$ on the versor);

(7) Generate a normalized vector $\mathbf{b}$ orthogonal to both $\mathbf{a}$ and $\mathbf{v}_{u}$ (e.g., with a vector product);

(8) Compute the post diffusion position as $\mathbf{v}_{f}=\mathbf{v}_{u}$ $+[\cos (\chi) \mathbf{a}+\sin (\chi) \mathbf{b}] \sin (u)$, where $\chi=2 \pi \xi$ is the polar angle measured from the plane defined by $\mathbf{v}_{u}$ and $\mathbf{a}$, and $\xi$ is an uniform random variate as above.

${ }^{1}$ A. Hernando, M. Barranco, R. Mayol, M. Pi, and F. Ancillotto, Phys. Rev. B 78, 184515 (2008)

${ }^{2}$ Y. Ren and V. V. Kresin, Phys. Rev. A 76, 043204 (2007).

${ }^{3}$ A. Przystawik, S. Göde, T. Döppner, J. Tiggesbäumker, and K.-H. MeiwesBroer, Phys. Rev. A 78, 021202(R) (2008).

${ }^{4}$ M. Mella, M. C. Colombo, and G. Morosi, J. Chem. Phys. 117, 9695 (2002).

${ }^{5}$ A. Wada, T. Takayanagi, and M. Shiga, J. Chem. Phys. 119, 5478 (2003).

${ }^{6}$ J. L. Persson, Q. Hui, Z. J. Jakubek, M. Nakamura, and M. Takami, Phys. Rev. Lett. 76, 1501 (1996).

${ }^{7}$ A. Bartelt, J. D. Close, F. Federmann, N. Quaas, and J. P. Toennies, Phys. Rev. Lett. 77, 3525 (1996)

${ }^{8}$ A. Przystawik, P. Radcliffe, S. G. Gode, K.-H. Meiwes-Broer, and J. Tiggesbäumker, J. Phys. B 39, S1183 (2006).

${ }^{9}$ S. A. Krasnokutski and F. Huisken, J. Phys. Chem. A 114, 7292 (2010).

${ }^{10}$ M. Mella, G. Calderoni, and F. Cargnoni, J. Chem. Phys. 123, 054328 (2005).

${ }^{11}$ S. Chiesa, M. Mella, G. Morosi, and D. Bressanini, J. Chem. Phys. 119, 5601 (2003)

${ }^{12}$ P. Håkansson, M. Mella, D. Bressanini, G. Morosi, and M. Patrone, J. Chem. Phys. 125, 184106 (2006).

${ }^{13}$ P. Håkansson and M. Mella, J. Chem. Phys. 126, 104106 (2007).

${ }^{14}$ K. Sakkos, J. Casulleras, and J. Boronat, J. Chem. Phys. 130, 204109 (2009).

${ }^{15}$ R. E. Zillich, J. M. Mayrhofer, and S. A. Chin, J. Chem. Phys. 132, 044103 (2010).

${ }^{16}$ M. Rossia, M. Nava, L. Reatto, and D. E. Galli, J. Chem. Phys. 131, 154108 (2009).

${ }^{17}$ M. W. Avilés and E. Curotto, J. Phys. Chem. A 111, 2610 (2007).

${ }^{18}$ D. C. Clary, J. Chem. Phys. 114, 9725 (2001).

${ }^{19}$ A. Sarsa, K. E. Schmidt, and J. W. Moskowitz, J. Chem. Phys. 113, 44 (2000).

${ }^{20}$ F. Russo Jr., and E. Curotto, J. Chem. Phys. 118, 6808 (2003).

${ }^{21}$ T. F. Miller and D. C. Clary, J. Chem. Phys. 116, 8262 (2002).

${ }^{22}$ E. Curotto, J. Chem. Phys. 123, 134102 (2005).

${ }^{23}$ V. Buch, J. Chem. Phys. 97, 726 (1992).

${ }^{24}$ R. A. Kuharski and P. J. Rossky, J. Chem. Phys. 82, 5164 (1985).

${ }^{25}$ A. Viel, M. V. Patela, P. Niyaza, and K. B. Whaley, Comput. Phys. Commun. 145, 24 (2002).

${ }^{26}$ T. Luan, E. Curotto, and M. Mella, J. Chem. Phys. 128, 164102 (2008).

${ }^{27}$ J. Faraudo, J. Chem. Phys. 116, 5831 (2002).

${ }^{28}$ R. Holysr, D. Plewczyǹski, and A. Aksimentiev, Phys. Rev. E 60, 302 (1999).

${ }^{29}$ L. Brualla, K. Sakkos, J. Boronat, and J. Casulleras, J. Chem. Phys. 121, 636 (2004)

${ }^{30}$ E. Curotto, Stochastic Simulation of Clusters (CRC, New York, 2009).

${ }^{31}$ M. H. Kalos, D. Levesque, and L. Verlet, Phys. Rev. A 9, 2178 (1974).

${ }^{32}$ F. Cargnoni and M. Mella, J. Phys. Chem. A 115, 7141 (2011).

${ }^{33}$ K. T. Tang, J. P. Toennies, and C. L. Yiu, Phys. Rev. Lett. 74, 1546 (1995).

${ }^{34}$ G. C. Groenenboom and I. M. Struniewicz, J. Chem. Phys. 113, 9562 (2000) 
${ }^{35}$ D. Bressanini, M. Zavaglia, M. Mella, and G. Morosi, J. Chem. Phys. 112, 717 (2000).

${ }^{36}$ P. Slaviček and M. Lewerenz, Phys. Chem. Chem. Phys. 12, 1152 (2010).

${ }^{37}$ A. Nakayama and K. Yamashita, J. Chem. Phys. 112, 10966 (2000).

${ }^{38}$ E. Coccia, E. Bodo, F. Marinetti, F. A. Gianturco, E. Yildrim, M. Yurtsever, and E. Yurtsever, J. Chem. Phys. 126, 124319 (2007).

${ }^{39}$ S. Moroni and S. Baroni, Phys. Rev. Lett. 24, 4745 (1999).

${ }^{40}$ S. Paolini, S. Fantoni, S. Moroni, and S. Baroni, J. Chem. Phys. 123, 114306 (2005).

${ }^{41}$ T. Ŝkrbić, S. Moroni, and S. Baroni, J. Phys. Chem. A 111, 12749 (2007).

${ }^{42}$ J. Tang, A. R. W. McKellar, F. Mezzacapo, and S. Moroni, Phys. Rev. Lett. 92, 145503 (2004).
${ }^{43}$ S. Moroni, M. Botti, S. De Palo, and A. R. W. McKellar, J. Chem. Phys. 122, 094314 (2005).

${ }^{44}$ S. Moroni, A. Sarsa, S. Fantoni, K. E. Schmidt, and S. Baroni, Phys. Rev. Lett. 90, 143401 (2003).

${ }^{45}$ S. Moroni, N. Blinov, and P.-N. Roy, J. Chem. Phys. 121, 3577 (2004).

${ }^{46}$ W. Topic, W. Jger, N. Blinov, P.-N. Roy, M. Botti, and S. Moroni, J. Chem. Phys. 125, 144310 (2006).

${ }^{47}$ E. Lugovoj, J. P. Toennies, and A. Vilesov, J. Chem. Phys. 112, 8217 (2000).

${ }^{48}$ C.-F. Nien, B. Rajasekhar, and J. M. C. Plane, J. Chem. Phys. 97, 6449 (1993). 